\title{
Barth Syndrome
}

National Cancer Institute

\section{Source}

National Cancer Institute. Barth Syndrome. NCI Thesaurus. Code C84585.

A rare $X$-linked syndrome caused by mutations in TAZ1 gene. Signs and symptoms

include cardiomyopathy, neutropenia, muscle weakness and atrophy, growth delay, cardiolipin deficiency and 3-methylglutaconic aciduria. 